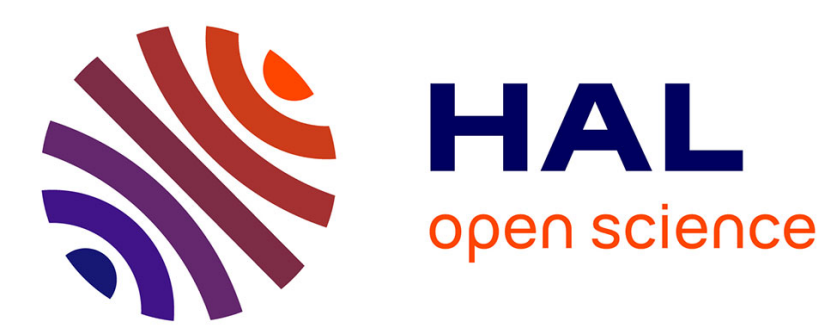

\title{
The sonorous beat of optical waves in a Michelson interferometer
}

\author{
Matthias Büchner, Jean-Philippe Loisel
}

\section{To cite this version:}

Matthias Büchner, Jean-Philippe Loisel. The sonorous beat of optical waves in a Michelson interferometer. American Journal of Physics, 2020, 88 (8), pp.612-616. 10.1119/10.0001208 . hal-02904427

\section{HAL Id: hal-02904427 \\ https://hal.science/hal-02904427}

Submitted on 26 Jul 2020

HAL is a multi-disciplinary open access archive for the deposit and dissemination of scientific research documents, whether they are published or not. The documents may come from teaching and research institutions in France or abroad, or from public or private research centers.
L'archive ouverte pluridisciplinaire HAL, est destinée au dépôt et à la diffusion de documents scientifiques de niveau recherche, publiés ou non, émanant des établissements d'enseignement et de recherche français ou étrangers, des laboratoires publics ou privés. 


\title{
The sonorous beat of optical waves in a Michelson interferometer
}

\author{
Matthias Büchner ${ }^{1, \text { a) }}$ and Jean-Philippe Loisel ${ }^{1}$ \\ Laboratoire Collisions Agrégats Réactivité-IRSAMC \\ Université de Toulouse III Paul Sabatier, CNRS UMR 5589, 118 Route de Narbonne, 31062 Toulouse, \\ France
}

(Dated: 26 July 2020)

This paper describes time dependant phase modulation experiments using an optical Michelson interferometer. Changes in the path length of the interferometer arms induce phase shifts in time and, thanks to a loudspeaker, the interferometric signal is audible. We produce time fluctuating refractive indices and mechanical motions of one reflecting mirror. A sinusoidal motion is applied, thus creating a phase modulation of one interferometer arm wave. Sidebands of the laser frequency appear which interfere with the other interferometer wave. Our hearing sense is quite developed and we use it to analyse the non linear character by the acoustic timbre. Finally we transfer information by phase modulation. Our set-up benefits to the visually-impaired community as temporal phase changes can be analysed by the auditory sense, but this unconventional use of an optical interferometer also attracts a general audience.

\section{INTRODUCTION}

The Michelson interferometer is a standard instrument to demonstrate interference of an optical wave. It can also be used as a precision instrument to detect tiny changes in the length of optical interferometer paths. One or both interferometer output beams can be sent to photodetectors and the optical intensity signals can be recorded, allowing high precision measurements of laser wavelengths $^{1,2}$ and distance changes ${ }^{3}$. One impressive example is the detection of gravitational waves with the LIGO experiment, rewarded in 2017 by the Nobel prize for R. Weiss, B.C. Barish and K.S. Thorne ${ }^{4-6}$,

Traditionally in education, the fringe patterns are observed visually and indeed their beauty is impressive and flawless.

Such an interferometer can also be used to demonstrate interferences in time, i.e. beats of optical waves. Mickey McDonald et al. ${ }^{7}$ described a Mach-Zehnder interferometer with an acousto-optical modulator (AOM), placed in each interferometer arm. These AOM shift differently the laser frequency by about $80 \mathrm{MHz}$ and their frequency difference is some Hertz. The optical beating of the two laser waves can be directly observed: the intensity at any point within the pattern is seen to oscillate sinusoidally in magnitude. The beauty of this experiment is the observation by eye of a pulsation in the Hertz region, at low frequencies never directly introduced in the experiment.

Our approach also aims to observe beat notes of optical waves, but our set-up places the beat observation into the sound world. In fact, our auditory sense ${ }^{8-12}$ can discern precise frequencies: our fascination with music is based on this fact ${ }^{13,14}$. Our set-up doesn't need AOMs and their highly stabilised power supplies. The use of the auditory sense is of interest for the visually-impaired community but also adds an amusing educational aspect for a wide-spread audience.

\footnotetext{
${ }^{a)}$ Electronic mail: matthias.buchner@irsamc.ups-tlse.fr
}

We add another component to a standard optical Michelson interferometer: we monitor the optical interference signal by a photodiode and we send its amplified signal to a loudspeaker or headphone. We placed one mirror on a piezo actuator controlled translation stage. Our goal is to detect relative optical path length changes; i.e. phase shifts in time, by simply listening to the sound.

Changing optical path length in time can be created by moving the position of the piezo-mounted mirror. Another possibility is to alter the refractive index of the optical medium probed by one interferometer arm. In our case, the optical medium is air, its pressure and temperature fluctuations change the optical path difference thus the signal amplitude. Interference in time, i e. beating of the optical waves takes place and thanks to our set-up this beat is audible.

In the following, we recall briefly the theoretical background. The experimental set-up will be described. We will then detail some experiments :

1. Touching the mirror mounts. This induces tiny changes in the mechanical path length difference, i.e. phase shifts in time which can be heard.

2. Making audible temporal changes of the refractive index of the air.

3. Applying a sinusoidal phase modulation by modulating the position of one mirror. Harmonics are created which depend on the motion amplitude. The order and intensity of the harmonics can be analysed by listening to the sound as the acoustical timbre changes.

4. Finally the electrical signal of a MP3 player is amplified and sent to a piezo mounted mirror, acting on the mirror position: The sound is transmitted by phase modulation and the interference signal contains this information.

Finally we will give hints to lower the costs, i.e. to replace some commercial material by low-cost home made components. A conclusion completes our paper. 


\section{THEORETICAL BACKGROUND}

We assume, that the light source emits a plane wave, with frequency $\nu_{L}$ and wavelength $\lambda_{L}$. The laser wavelength bandwidth is supposed to be negligibly small. The electric field is then given by the well-known equation:

$$
\begin{aligned}
E(t, x) & =E_{0} \cos \phi(x, t), \text { with } \\
\phi(x, t) & =2 \pi\left[\frac{x}{\lambda_{L}}-\nu_{L} t\right]
\end{aligned}
$$

The incident wave is split into two parts with equal intensities (see fig.1). We use a 50\%-50\% beamsplitter, its coated side points to the right. One wave is travelling to the right; the other is reflected at the second (coated) surface of the beamsplitter and travels upwards. Both beams are reflected by two mirrors and are superposed on the beam splitter, which recombines both beams. The upper wave is phase shifted by $4 \pi L / \lambda_{L}$, where $L$ is the distance between the beam splitter and the mirror. A phase shift $\varphi_{1}$ is added to include other differences in the optical path, e.g. due to perturbation of the air refractive index. The other wave undergoes a similar phase shift of $4 \pi(L+\Delta x) / \lambda_{L}+\varphi_{2}$, where $L+\Delta x$ is the distance between the right mirror and the beamsplitter.

The wave ("1") travelling downwards passes through the beam splitter, while the other wave ("2") is reflected at the right (air-side) surface, undergoing a $\pi$ phase shift. We dropped all phase shifts related to the beamsplitter thickness. Both waves are superposed and form an output beam, situated at the lower part where we place our detector. Defining the wave phases $\phi_{1(2)}$ at the detector position $y_{D}$ :

$$
\begin{aligned}
& \phi_{1}=2 \pi\left(y_{D}+2 L\right) / \lambda_{L}+\varphi_{1}-2 \pi \nu t \\
& \phi_{2}=2 \pi\left(y_{D}+2 L+2 \Delta x\right) / \lambda_{L}+\varphi_{2}+\pi-2 \pi \nu t
\end{aligned}
$$

We can write $\tilde{E}(t)^{2}=\left[E\left(t, y_{D}\right) / E_{0}\right]^{2} / 2$ :

$$
\begin{aligned}
\tilde{E}(t)^{2} & =\left[\cos \phi_{1}(t)+\cos \phi_{2}(t)\right]^{2} \\
& =\cos ^{2} \phi_{1}+\cos ^{2} \phi_{2}+2 \cos \phi_{1} \cos \phi_{2} \\
& =\cos ^{2} \phi_{1}+\cos ^{2} \phi_{2}+\cos \left(\phi_{1}-\phi_{2}\right)+\cos \left(\phi_{1}+\phi_{2}\right)
\end{aligned}
$$

The detected intensity, $I(t)$, is proportional to $\left\langle E\left(t, y_{D}\right)^{2}\right\rangle_{T}$, where $\langle.\rangle_{T}$ stands for the average over the detector response time $\mathrm{T}$ with $T>2 \pi / \nu_{L}$. The terms $\left\langle\cos ^{2} \phi_{1(2)}\right\rangle_{T}$ are oscillating at twice the optical frequency $\nu_{L}=474 \mathrm{THz}\left(\lambda_{L}=632.8 \mathrm{~nm}\right)$, and each term averages to $1 / 2$. For the same reason, the interference term $\left\langle\cos \left(\phi_{1}+\phi_{2}\right)\right\rangle_{T}$ vanishes. We get

$$
\begin{aligned}
I(t) & \propto 1+\cos \left(\phi_{1}-\phi_{2}\right) \\
& \propto 1+\cos \left(4 \pi \Delta x / \lambda_{L}+\varphi_{2}-\varphi_{1}\right)+\pi
\end{aligned}
$$

\section{EXPERIMENTAL SET-UP}

Figure 1 shows our set-up. The optical part is the well know Michelson interferometer. An initial wave is emitted by the He-Ne laser (Lasos LGR7621, 5mW, $\lambda_{L}=632.8$ $\mathrm{nm})$.

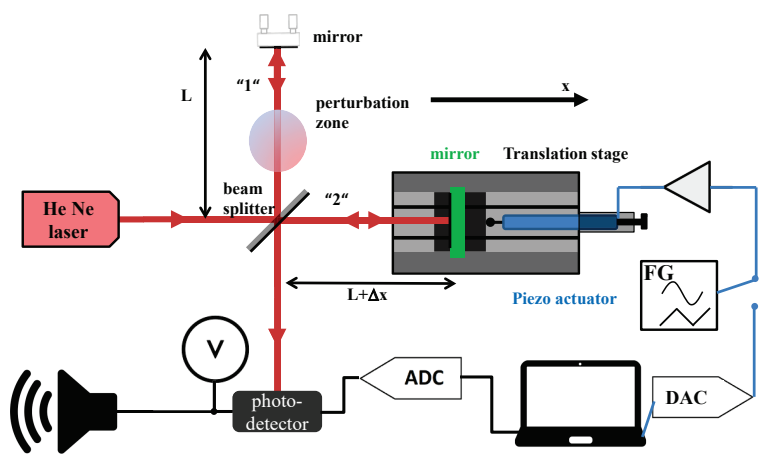

FIG. 1. Experimental set-up of our Michelson interferometer. One mirror is mounted on a piezo-driven translation stage and the interference signal is monitored by a photodetector. The photodetector signal is amplified and sent to a loudspeaker and to a computer via an analog-digital convertor (ADC). A pilot voltage is created by summing up a DC bias and a time varying voltage. This pilot voltage is amplified and drives the piezo actuator mounted mirror thus changing the optical path length. This pilot voltage can be created by a function generator (FG), a digital-analog convertor (DAC) or a MP3 player.

A beam splitter separates this wave into two equal parts, one travelling to the right, the other upwards. Thanks to two mirrors (Thorlabs PF10-03-P01), both waves are reflected and superposed on the beam splitter.

The beam splitter recombines the two waves and two output beams can be distinguished, one at the bottom of the figure, the other on the left, with the outgoing beam pointing towards the laser. Remember, that this outgoing beam should not be injected into the laser. Large fluctuations in intensity and frequency or even laser damage may occur otherwise.

We monitor the first output beam using the amplified signal from a photodetector (Thorlabs PDA36A2). The signal is connected to a oscilloscope, a loudspeaker and to a computer via an analog-digital convertor. We used the software Labview (National Instruments) to communicate with a multifunction card (National Instruments: PCI 6259).

The right mirror is mounted on a commercial translation stage (ACMEL TM-21-10). A piezo actuator (Piezomechanik GmbH Pst 150/5/40) with a maximum extension of $40 \mu \mathrm{m}$ was placed between the moving element and an end-mounted positioning screw. We use a low voltage which is composed by a DC bias and a time varying voltage. This pilot voltage is amplified by a homemade amplifier up to $100 \mathrm{~V}$ to drive the piezo actuator. The choice of all components was dictated by the availability of the material in our laboratory.

At the beginning, it is convenient to align the Michelson interferometer as usual, i.e. in the traditional optical way. Using a ruler, we equalize the distances between the mirrors and the beamsplitter. We superposed the 
two outgoing beams till fringes are visible. Then, we act carefully on the inclinations of the mirrors to get the fringes as big as possible. The observed signal of the photodetector is given by:

$$
I(t)=I_{0}\left[1+\mathcal{V} \cos \left(\frac{4 \pi \Delta x(t)}{\lambda_{L}}+\varphi_{2}-\varphi_{1}\right)\right]
$$

with $I_{0}$ the mean value, proportional to the laser power, and $\Delta x(t)$ the optical path length difference of the interferometer arms. This difference can be varied by the piezo actuator. $\mathcal{V}$ is the fringes visibility with $\mathcal{V} \leq 1$. There are many reasons which lead to smaller $\mathcal{V}$, we can cite non-perfect superposition of the interfering beams and a path difference longer than the coherence length.

Once fringes are visible, a triangular signal from a waveform generator drives the position of the mounted mirror in a linear forth-back motion. The photodetector signal is monitored by an oscilloscope. We used the modulation signal to improve the alignment.

Typical values for our Michelson interferometer are $\mathcal{V}=95 \%$ and $I_{0}=4.5 \mathrm{~V}$. Our maximal phase sensitivity $\Delta I / \Delta \phi=I_{0} \mathcal{V}$ is about $4.3 \mathrm{mV} / \mathrm{mrad}$ and for the displacement sensitivity we get $\Delta I / \Delta x=85 \mathrm{mV} / \mathrm{nm}$.

$I(t)$ contains two parts (see eq.(4)). The constant part, $I_{0}$, can not be heard. The other part is proportional to the fringe visibility $\mathcal{V}$ and $I_{0}$ and this part produces the sound. For typical speeds of the mirror mount around $v_{M}=70 \mu \mathrm{m} / \mathrm{s}, \nu_{M}$ is about $221 \mathrm{~Hz}$ and this interference signal can be heard thanks to the loudspeaker.

\section{APPLICATIONS}

In this section, we will discuss several applications of our set-up and detail the underlying physics. The first two and the last examples (subsections $\mathrm{A}, \mathrm{B}, \mathrm{D}$ ) concern qualitative experiments, while $\mathrm{C}$ is of more quantitative nature.

\section{A. Transforming mechanical motion into sound: playing like a disk-jockey}

A "starter" to demonstrate the sensitivity of the interferometer is to transform mechanical vibration into sound. By changing the DC bias voltage, we set our interferometer to work around mid-fringe, i.e. at maximum phase sensitivity. Just by pushing gently on the fixed mirror, one interferometer path length is changed in time, the fringes move and you can create sounds in a disk-jockey way. i.e. moving vinyl discs by hand back and forth.

The file "disk-jockey.wav" ${ }^{15}$ contains one example of sound recording. During the first 7 seconds, one can hear the vibrations induced by the footsteps. Then, the fixed mirror is pushed and the time varying path length difference leads to intensity fluctuation, thus to audible sounds.
One might think, that the pushed mirror changed the direction of the laser beam and only a fraction of the laser beam hit the sensitive area of the photodetector thus inducing a different signal. While continuing the pushing, we blocked at 14 seconds the right interferometer arm: no sounds are created. Unblocking this interferometer arm, again the sound is audible. We now blocked the other arm and again the sound died. This experiment proves that both interferometer arms are needed to create sounds. We also pushed the other mirror with the same results (29 seconds).

An alternative approach is to reduce the visibility to zero by misaligning the interferometer. The resulting signal is given only by $I_{0}$ and no sounds are audible.

\section{B. Listening to refractive index fluctuations}

We can induce refractive index fluctuations on one interferometer arm. The refractive index of air depends on the temperature and the pressure. Taking the equation of Birch and Downs ${ }^{16}$ for dry air at temperature $20^{\circ} \mathrm{C}$ and pressure 1 bar $(100 \mathrm{kPa})$, a pressure change of +10 $\mathrm{kPa}$ increases the refractive index of $\Delta n \approx 3 \times 10^{-5}\left(\lambda_{L}=\right.$ $633 \mathrm{~nm})$. Over a length of $\ell=1 \mathrm{~mm}$, this change induces a phase shift of $\Delta \varphi=2 \pi \Delta n\left(\ell / \lambda_{L}\right) \approx 0.3 \mathrm{rad}$. For a temperature change of $+10{ }^{\circ} C$, we get $\Delta n \approx-0.9 \times 10^{-5}$, i.e. $\Delta \varphi \approx-0.1 \mathrm{rad}$.

We provoked air density fluctuations in the probe zone. We tried to apply a heating resistance under one interferometer arm, the fringes did move but their motion was too slow to be audible. Better results were achieved with a lightened match, although not to be recommendable nearby optical elements ("allumette.wav"15). We employed an air duster with good results. A weak air stream is directed towards one interferometer beam in a vertical direction.

The file "airduster.wav"15 contains the sound produced (start at 4 seconds). At 11 seconds, one interferometer arm is blocked, released and at 14 seconds the other arm is blocked. Of course, both interferometer beams should be present to produce the sound. At 19 seconds the air stream is directed towards the blind side of the mirror mount. No sound can be heard, i.e. the sound heard before is not related to a hypothetical mirror vibration produced by the air stream.

\section{Generation of frequency harmonics}

Our set-up can be used to create frequency combs of the main laser frequency. Theodor W. Hänsch ${ }^{17}$ and John Hall ${ }^{18}$ received the Nobel prize in 2005 for their ground breaking work on laser frequency combs and their important applications in high resolution spectroscopy.

We create frequency combs by phase-modulating one optical wave in the interferometer. A sinusoidal pilot voltage is applied to the piezo actuator, i.e. the mirror 
is oscillating with an amplitude $a_{m}$ and frequency $\nu_{m}$ : $\Delta x=\Delta x_{0}+a_{m} \sin \left(\omega_{m} t\right)$, with the angular frequency $\omega_{m}=2 \pi \nu_{m}$. By changing the DC bias voltage, $\Delta x_{0}$ can be varied slowly in time. Inserting the fast sinusoidal motion, eq. (4) reads:

$$
\begin{aligned}
I(t) & =I_{L}\left[1+\mathcal{V}\left(\cos \left(\varphi_{0}+\varphi_{m} \sin \left(\omega_{m} t\right)\right)\right)\right] \\
& \left.=I_{L}\left[1+\mathcal{V} \cos \varphi_{0}\left(\mathcal{J}_{0}\left(\varphi_{m}\right)+2 \sum_{k=1}^{\infty} \mathcal{J}_{2 k}\left(\varphi_{m}\right) \cos \left(2 k \omega_{m} t\right)\right)\right)-\mathcal{V} \sin \varphi_{0}\left(\sum_{k=1}^{\infty} 2 \mathcal{J}_{2 k-1}\left(\varphi_{m}\right) \sin \left((2 k-1) \omega_{m} t\right)\right)\right]
\end{aligned}
$$

with $\varphi_{0}=4 \pi \Delta x_{0} / \lambda_{L}, \varphi_{m}=4 \pi a_{m} / \lambda_{L}$. We developed $I(t)$ using first-order Bessel functions $\mathcal{J}_{k}$ (see e.g. ref. ${ }^{19}$ ).

Harmonics of the mirror excitation frequency $\nu_{m}$ are created with frequencies and amplitudes governed by $\mathcal{J}_{k}$. If we choose $\varphi_{0}=-\pi / 2$, the total phase is modulated around zero, i.e. at midfringe. Only odd harmonics exist. For $\varphi_{0}=0$, modulation occurs at the maximum intensity. Only even harmonics exist.

We recorded the interference signal of a phase modulation with a frequency $\omega_{m}=2 \pi \times 220 \mathrm{~Hz}$ and a small modulation amplitude of $\varphi_{m}=0.14 \mathrm{rad}$. Figure 2 shows our data.

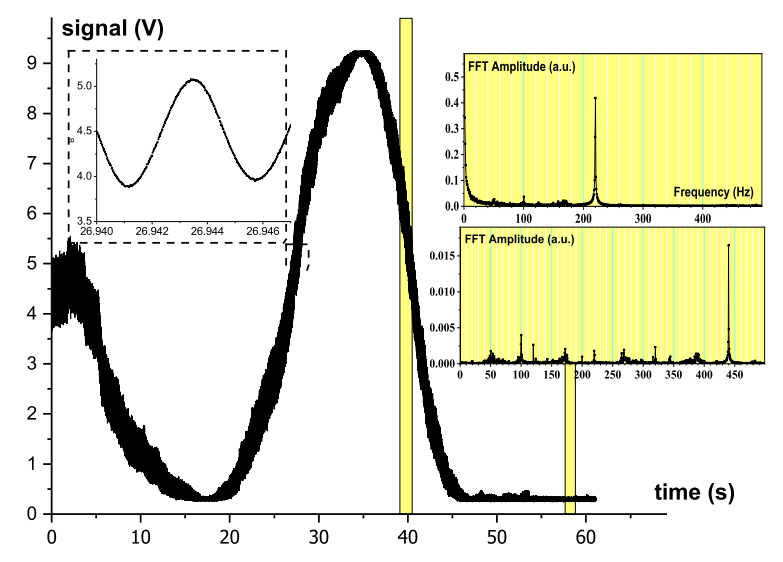

FIG. 2. A sinusoidal phase modulation with $\varphi_{m}=0.14$ and $\omega_{m}=2 \pi \times 220 \mathrm{~Hz}$ was applied on one interferometer arm. We varied slowly $\varphi_{0}$ as a function of time. The left hand inset shows a zoom on the recorded data demonstrating the applied modulation. A subset of data (inside the rectangles at 39 and $58 \mathrm{~s}$ ) was selected for a Fast Fourier transform (FFT) analysis. The results are shown in the right hand insets.

We varied slowly the offset of the DC bias voltage, thus scanning the interference fringes. We pass from midfringe through the minimum (17 s recording time) and the maximum intensity (34 s). For this small phase modulation amplitude only the fundamental $(k=1)$ and the first harmonic $(k=2)$ contribute to the signal: $\mathcal{J}_{1}(0.14)=0.06983(220 \mathrm{~Hz})$ and $\mathcal{J}_{2}=0.00245(440 \mathrm{~Hz})$.

The rectangles on the graph enclose the data subsets taken for two Fourier analysis shown on Figure 2. The upper FFT graph corresponds to data in the mid-fringe window ( $\approx 39 \mathrm{~s}$ ) and the lower to data around minimum $(\approx 58 \mathrm{~s})$. The first FFT analysis shows the presence of the fundamental frequency only $(220 \mathrm{~Hz})$ while the second exhibits the second harmonic $(440 \mathrm{~Hz})$ only. As expected, these results are in complete agreement with the Bessel function amplitude.

We converted the data to a sound file ("220Hz10mV.wav"15). While scanning the pilot offset voltage, the change in timbre can easily be recognized. At mid-fringe $\left(\varphi_{0}=-\pi / 2,28\right.$ s recording time) the fundamental at $220 \mathrm{~Hz}$ (music notation: $A_{3}$ ) dominates as expected, while for the minima and maxima the second harmonic $\left(440 \mathrm{~Hz}, A_{4}, 17,34\right.$ and $\left.47 \mathrm{~s}\right)$, can clearly be heard. The $440 \mathrm{~Hz}$ sound component has lower volume, about $4 \%$ of the $220 \mathrm{~Hz}$ one, according to the amplitude ratio $\left|\mathcal{J}_{2}(0.14) / \mathcal{J}_{1}(0.14)\right|$.

For the following experiment we set the offset of the pilot function to mid-fringe, i.e. $\varphi_{0}=\pi / 2$. We applied a $220 \mathrm{~Hz}$ modulation changing the modulation amplitude pilot voltage in order to vary the phase modulation amplitude $\varphi_{m}$ from 0.25 to $7.5 \mathrm{rad}$ in steps of 0.25 . The file "midfringes3.wav" contains the recorded sound.

At the beginning the volume of the fundamental frequency increases with higher modulation amplitudes. While increasing $\varphi_{m}$, the change in timbre can clearly be recognized. In fact the harmonics get stronger. At 24 seconds the third harmonic $\left(660 \mathrm{~Hz}, A_{5}\right)$ can be distinguished, but this depends on the person listening.

However, one has to be aware that $\varphi_{0}$ drifts slowly as we do not employ any active stabilisation techniques. Additionally, the response of a piezo actuator is non linear to the applied voltage and this is particularly true for large voltage amplitudes. As a consequence, the phase modulation is only approximately linear.

At 49 seconds of recording time, the $220 \mathrm{~Hz}$ sound is weak. This corresponds to the first zero of the Bessel function $\mathcal{J}_{1}\left(\varphi_{m}\right)$ with $\varphi_{m}=3.8 \mathrm{rad}$. Using the first part of eq.(5), we fitted the interference signal and got $\varphi_{m}=$ $3.8 \mathrm{rad}$, which is in agreement with the theoretical value. For high phase amplitude modulations, higher order harmonics are produced, we can speak of a frequency comb.

This frequency comb has frequency spacing in the 1002000 Hertz region, well below the typical bandwidth of 
a multimode $633 \mathrm{~nm}$ free running He-Ne laser, typically in the $\mathrm{GHz}$ region. As a consequence, sidebands can not be resolved in the energy (frequency) spectrum, but as we superpose this frequency comb to a coherent reference wave (the other interferometer arm), frequency fluctuations of the laser source are rejected and beats are produced which are in our case audible. This is the base of homodyne detection technique.

\section{Transfer of music by phase modulation of optical waves}

An interesting experiment is the transmission of music by phase modulation of optical waves. We adjust our interferometer to mid fringe $\varphi_{m}=\pi / 2$. We used a sound file with music ("04-petit-pantin-au-coeur-deglace-eponyme.wav" $\left.{ }^{26}\right)$. The sound amplitudes are converted by a Labview program and a digital-analog convertor produces the driving voltage for the piezo actuator, i.e. sound is converted to mechanical motion. The amplification and the sound volume should be weak in order to privilege the fundamental frequency in phase modulation. Higher amplitudes promote higher harmonics, thus distortion of the sound. The sound file "musicPM.wav" contains the interference signal.

During a demonstration, one person of the audience may be invited to use his/her mp3 player to plug the driving voltage input into the sound port. The sound is transmitted via phase modulation and this can be verified by blocking the interferometer arms one after the other: once again the need of two laser beams for the transmission is demonstrated.

A discussion may be triggered about the advantages and inconveniences of the optical phase modulation techniques. Obviously this transmission protocol is not very handy for every-day use as this setup is very sensitive to vibrations and two coherent laser beams are needed (the two interferometer arms). However phase-modulation, in a radio-frequency or optical domain, is very important for research, we can cite the Pound-Drever-Hall technique for laser locking ${ }^{24,25}$.

According to quantum mechanics, phase modulation, especially beating (or the transmission of music), is not reserved to acoustic or electromagnetic waves: matter waves can also be phase modulated in time. We can cite the work of Décamps et al. ${ }^{21,22}$ (and refs. within). The group studied beating of lithium waves by phase modulation in an atom Mach-Zehnder interferometer and transmission of music has been realised by phase modulated atom waves (see Décamps et al. ${ }^{23}$ ).

\section{E. Reducing costs and improvements}

The He-Ne laser can be replaced by a low cost laser pointer. The replacement of the electronics and/or the mechanical parts depends on the skills in electronics and mechanics of the user: the high voltage amplification unit and the photodetector may be replaced by home-made versions as well as the mirror mountings. In fact, the pilot voltage is less than $10 \mathrm{~V}$ and it must be biased by a static voltage of about $50 \mathrm{~V}$. The piezo actuator can be replaced by an positioner based on electromagnetic forces. Yin-Yen Kuo et al. ${ }^{27}$ describe a 3D printed positioner which has micrometer accuracy. The displacement of a permanent magnet is controlled by a coil and the magnet acts on a moving stage.

\section{v. CONCLUSION}

We used an optical Michelson interferometer to make time-varying optical path length changes audible. Our hearing, especially recognizing frequency changes, is very sensitive and we use it to demonstrate beat notes of optical waves. We apply this interferometer to recognize mechanical vibration of the interferometer mirrors and refractive index fluctuations of the air.

We applied a sinusoidal phase modulation by mechanically displacing one interferometer mirror. The nonlinear response in phase of the interferometer leads to the creation of harmonics of the fundamental frequency. Optical frequency combs can be created, their beat has timbres as a function of applied phase modulation amplitude. Finally we realized a transmission of music by phase modulation of an optical wave.

This non-conventional set-up adds educational aspects, which are original and amusing. Making optical interference audible paves the way for a visually-impaired community to understand interferences of optical waves and but we think that the audible character of interferences also interests a general audience. The "disk-jockey" experiment has already been shown during a public event in our university. The audience comprised pupils of secondary education level. The experiments and the underlying physics described in this paper will serve for educational events, courses at school and talks for general public.

\section{ACKNOWLEDGMENTS}

We gratefully acknowledge Laurent Polizzi, Eric Panader and Michel Gianesin for the realisation of some mechanical supports. The authors owe particular gratitude to Bruno Lepetit and Stephen Gibson for fruitful discussions and careful review of the manuscript. Financial support from the INP-CNRS and the Université Toulouse III Paul Sabatier is acknowledged.

\footnotetext{
${ }^{1}$ M. Dobosz, M. Kożuchowski, "Overview of the laser-wavelength measurement methods", Optics and Lasers in Engineering 98 10711 (2017)

${ }^{2}$ J. Pedregosa-Gutierrez, D. Guyomarch, M. Vedel, C. Champenois and M. Knoop, "Computer-controlled high-precision Michelson wavemeter", Eur. Phys. J. Plus 129203 (2014)
} 
${ }^{3}$ K. Riles, "Gravitational waves: Sources, detectors and searches", Prog. Part. Nucl. Phys. 68, 1-54 (2013)

${ }^{4} \mathrm{R}$. Weiss, "LIGO and the Discovery of Gravitational Waves, I: Nobel Lecture, December 8, 2017“, Ann. Phys. 531, 1800349 (2019)

${ }^{5}$ B.C. Barish, "LIGO and Gravitational Waves II: Nobel Lecture, December 8, 2017", Ann. Phys. 531, 1800357 (2019)

${ }^{6}$ K.S. Thorne, "LIGO and Gravitational Waves, III: Nobel Lecture,December 8, 2017 “, Ann. Phys. 531, 1800350 (2019)

${ }^{7}$ Mickey McDonald, Jiyoun Ha, Bart H. McGuyer, and Tanya Zelevinsky, "Visible optical beats at the hertz level", American Journal of Physics 82, 1003-1007 (2014)

${ }^{8}$ G. Stix, "How Hearing Works [Video]" video published by Scientific American, August 1 (2015): "www.scientificamerican.com/article/how-hearing-works-video"

${ }^{9} \mathrm{~S}$. Rigby, "Human brains naturally tuned to hear music", Science Focus, 20th July (2019),

"https://www.sciencefocus.com/news/human-brains-naturallytuned-to-hear-music/"

${ }^{10}$ Joseph E. Hawkins, "Human ear", in Encyclopædia Britannica inc, October 25 (2018),

"www.britannica.com/science/ear"

${ }^{11}$ W.M. Hartmann, "Signals, Sound, and Sensation ", Springer Science \& Business Media, Springer, New York, NY (2004)

${ }^{12}$ C.J. Plack, "The Sense of Hearing", 3rd Edition, London: Routledge (2018)

${ }^{13}$ O. Freke, "Sine language", in Plus Magazine, University of Cambridge, Millennium Mathematics Project, Centre for Mathematical Sciences, March 1 (2009),

"plus.maths.org/content/sine-language"

${ }^{14}$ W.M. Hartmann , "Principles of Musical Acoustics", Springer Science+Business Media, Springer, New York, NY (2013)

${ }^{15}$ supplementary material, [URL will be inserted by AIP]

${ }^{16}$ K.P Birch and M.J Downs, "An Updated Edlén Equation for the
Refractive Index of Air", Metrologia 30, 155-162 (1993)

${ }^{17}$ Theodor W. Hänsch, "Nobel Lecture: Passion for precision", Rev. of Modern Physics, 78, 1297-1309 (2006)

${ }^{18}$ John L. Hall, "Nobel Lecture: Defining and measuring optical frequencies", Rev. of Modern Physics, 78, 1279-1295 (2006)

${ }^{19}$ M. Abranowitz and I.A. Stegan, "Handbook of Mathematical Functions: with Formulas, Graphs, and Mathematical Tables", Dover Publications, Inc. New York, $9^{t h}$ edition (1965)

${ }^{20}$ R. Feynman, R. B. Leighton, and M. Sands, "The Feynman Lectures on Physics", (Addison-Welsley, Reading, MA,1962), Vol. I, 2nd printing (1966)

${ }^{21}$ B. Décamps et al., "Observation of Atom-Wave Beats Using a Kerr Modulator for Atom Waves ", Phys. Ref. Lett. 116, 053004053004-5 (2016)

${ }^{22} \mathrm{~B}$. Décamps et al., "Phase modulation of atom waves: theory and experiment using the atom optics analogue of the Kerr effect", Europ. Journal of Physics D 71, 334 (2017)

${ }^{23} \mathrm{~B}$. Décamps et al., "Observation of Atom-Wave Beats Using a Kerr Modulator for Atom Waves “, Phys. Ref. Lett. 116, 053004053004-5 (2016), suppl. material

${ }^{24}$ R.W.P. Drever, J.L. Hall, J. L.; F.V. Kowalski, J. Hough, G.M. Ford, A.J. Munley, H. Ward, "Laser phase and frequency stabilization using an optical resonator"; Applied Physics B. 31, 97-105 (1983)

${ }^{25}$ Eric D. Black, "An introduction to PoundDreverHall laser frequency stabilization", Am. J. Phys. 69, 79-87 (2001)

${ }^{26}$ Laei, "Petit pantin au coeur de glace", source: lacrymosa. tuxfamily.org/, Licence: artlibre.org/licence/lal, www. auboutdufil. com/index . php?id=458 (6MB)

${ }^{27}$ Y.-Y Kuo, C.-H; Cheng and Sh.-K. Hung, "3D-Printed Linear Positioner with Micrometer Accuracy “, MATEC Web of Conferences 95, 10005 (2017) 